\title{
Ambiente obesogênico: uma cartografia do ambiente alimentar comunitário de uma capital do Nordeste
}

\author{
Obesogenic environment: cartography of a Brazilian Northeast capital
}

Ambiente obesogénico: una cartografía del ambiente alimentario comunitario de una capital del

Noreste de Brasil

Letícia Caroline da Silva Oliveira

ORCID: https://orcid.org/0000-0001-5103-0128 Universidade Federal de Sergipe, Brasil E-mail: oliveirallecca@gmail.com

Joana Carla Nunes de Souza

ORCID: https://orcid.org/0000-0001-5265-0716 Universidade Federal de Sergipe, Brasil E-mail: joanacarla.nutri@gmail.com

Fernanda Oliveira Souza

ORCID: https://orcid.org/0000-0001-7007-410X Universidade Federal de Sergipe, Brasil

E-mail: fosouza1@gmail.com.br

Marcela Larissa Costa

ORCID: https://orcid.org/0000-0002-2350-0193 Universidade Federal de Sergipe, Brasil E-mail: marcelaa.costa.nutricionista@gmail.com.br Raquel Simões Mendes-Netto

ORCID: https://orcid.org/0000-0001-8238-8958 Universidade Federal de Sergipe, Brasil

E-mail: raquel@academico.ufs.br

Diego Campana Loureiro

ORCID: https://orcid.org/0000-0003-0044-5280 Universidade Federal de Sergipe, Brasil E-mail: campanaloureiro@gmail.com Andhressa Araújo Fagundes

ORCID: https://orcid.org/0000-0003-4085-3270 Universidade Federal de Sergipe, Brasil E-mail: andhressa@academico.ufs.br

\begin{abstract}
Resumo
Estudo com o objetivo de quantificar e mapear os estabelecimentos comerciais alimentares e os locais para prática de atividade física de uma capital do nordeste do Brasil, visando analisar o ambiente alimentar obesogênico. Foi realizada pesquisa cartográfica, transversal, por geocodificação de endereços e coordenadas geográficas dos estabelecimentos de comercialização de alimentos e locais para prática de atividade física de toda a capital. Os estabelecimentos de alimentação foram classificados em segundo a oferta predominante de alimentos e/ou refeições, conforme o Guia Alimentar para a População Brasileira. Os locais para atividade física foram categorizados em públicos e privados. O mapeamento foi gerado por um Sistema de Informação Global. Foi realizada análise da densidade populacional, renda e distribuição espacial segundo o grau de aglomeração por zonas administrativas. Foram identificados 725 estabelecimentos de alimentação e 236 locais para a prática de atividade física, sendo 34,5\% estabelecimentos de alimentação saudáveis e $47,9 \%$ espaços públicos para atividade física. Os estabelecimentos não saudáveis (Grupo 3) estiveram mais presentes $(27,4 \%$ ) na zona de baixa densidade populacional e os estabelecimentos saudáveis (Grupos 1 e 2) mais presentes $(64 \%)$ na zona de alta renda domiciliar ( $<<0,01)$. As variáveis analisadas estiveram aglomeradas na zona Centro/Sul. Concluiu-se que a população está exposta tanto a um ambiente promotor da obesidade, quanto à alimentação saudável e aos locais para a prática de atividade física. Estratégias que incentivem e facilitem as escolhas saudáveis, e que protejam os indivíduos de fatores ambientais que conduzem às escolhas não saudáveis, podem ser tomadas por meio de políticas públicas.
\end{abstract}

Palavras-chave: Meio ambiente; Saúde pública; Obesidade; Mapeamento geográfico; Atividade física. 


\begin{abstract}
The aim of the study was to quantify and to map commercial food establishments and places for physical activity in a Brazilian Northeast capital, seeking to analyze the obesogenic food environment. A cartographic, cross-sectional survey was carried out by geocodification and geographic coordinates of food establishments and places for physical activity around the capital. The food establishments were classified according to their food supply and/or meal service, in conformity with the Dietary Guidelines for the Brazilian Population. The places for physical activity were categorized as public and private. The mapping was created by a Global Information System. Population density, income, and spatial distribution were analyzed according to the degree of agglomeration by administrative zones. 725 food establishments and 236 places for physical activity were identified, where $34.5 \%$ were classified as a healthy food establishment and $47.9 \%$ as public spaces for physical activity. Unhealthy food establishments are more present $(27.4 \%)$ in the low population density areas and healthy establishments are more present $(64 \%)$ in the high household income zone $(\mathrm{p}<0.01)$. The analyzed variables are clustered in the Central/South zone. It was concluded that the population is both exposed to an obesity-promoting environment, healthy food and places for physical activity. Strategies that encourage and facilitate healthy choices to protect individuals from unhealthy environmental factors and choices should be taken through public policies.
\end{abstract}

Keywords: Environment; Public health; Obesity; Geographic mapping; Physical activity.

\title{
Resumen
}

El trabajo busca cuantificar y mapear establecimientos comerciales de alimentos y lugares para la actividad física en una capital del noreste de Brasil, con el objetivo de analizar el ambiente alimentario obesogénico. Se realizó un levantamiento cartográfico transversal mediante geocodificación de direcciones y coordenadas geográficas de establecimientos comerciales de alimentos y lugares de actividad física en toda la capital. Los establecimientos de alimentos se clasificaron según la oferta predominante de alimentos y/o comidas, de acuerdo con la Guía de Alimentos para la Población Brasileña. Los espacios para la actividad física se categorizaron en públicos y privados. El mapeo fue generado por un Sistema de Información Global. Se realizó un análisis de densidad poblacional, ingresos y distribución espacial según el grado de aglomeración por zonas administrativas. Se identificaron un total de 725 establecimientos de alimentación y 236 lugares para la práctica de actividad física, siendo el 34,5\% establecimientos de alimentación saludable y el 47,9\% espacios públicos para la actividad física. Los establecimientos insalubres fueron más presentes en la zona de baja densidad poblacional y los establecimientos saludables están más presentes en la zona de altos ingresos. Las variables analizadas se agruparon en la zona Centro /Sur. Se concluyó que la población está expuesta tanto a un ambiente propicio para la obesidad, como a una alimentación saludable y lugares para la actividad física. Las estrategias que facilitan elecciones saludables y que protegen a las personas de los factores ambientales que conducen a elecciones poco saludables pueden adoptarse a través de políticas públicas.

Palabras clave: Medio ambiente; Salud pública; Obesidad; Mapeo geográfico; Actividad física.

\section{Introdução}

Diversos fatores influenciam as escolhas e o comportamento alimentar dos indivíduos, sendo estes também considerados determinantes de saúde. Esses determinantes podem ter um papel causal no aparecimento de doenças e deficiências, como também podem promover perspectivas de prevenção de doenças e manutenção da saúde, sendo que alguns deles explicam o aumento expressivo da obesidade na população (Cockerham et al., 2017; Paim, 2012). Sabe-se que a prevalência de obesidade vem crescendo em todos os países do mundo, sendo considerada nos dias atuais parte da sindemia global de obesidade, desnutrição e mudanças climáticas. (Swinburn et al., 2019).

A OMS estimou em 2016 uma prevalência global de obesidade de 13\% (World Health Organization, 2021). No Brasil, a Pesquisa Nacional de Saúde (PNS) de 2019 demonstrou uma prevalência de 25,9\% para obesidade (Instituto Brasileiro de Geografia e Estatística, 2020a), esses dados quando somados a prevalência de 40,3\% de inatividade física e o ao baixo consumo de frutas e vegetais e consumo elevado de alimentos ultraprocessados (Instituto Brasileiro de Geografia e Estatística, 2020b), fez com que os gastos com obesidade no Sistema Único de Saúde chegassem a 1,39 bilhão de reais/ano (Nilson et al., 2020). Essa alta prevalência de obesidade é explicada pobremente quando avaliada a nível individual, sendo assim se faz necessário avaliar também as influências sociais, políticas e ambientais neste processo (Glanz et al., 2005).

Em Aracaju, capital do estado de Sergipe, cerca de 20,6\% da população é obesa e 44,9\% é insuficientemente ativa (Brasil, 2020). O município conta com 571.149 habitantes e alta densidade populacional $\left(3.140,65\right.$ hab/ $\left./ \mathrm{km}^{2}\right)$. Tem um Índice 
de Desenvolvimento Humano de 0,77, considerado alto e superior à média nacional de 0,75 (Instituto Brasileiro de Geografia e Estatística, 2011). Em 2008 foi considerada a capital da qualidade de vida, ficando em primeira posição no ranking das capitais mais saudáveis do país, em pesquisa realizada pelo Ministério da Saúde (Prefeitura de Aracaju, 2008).

Essa elevada prevalência de obesidade é explicada de forma superficial quando avaliada a nível individual, sendo assim se faz necessário avaliar também as influências sociais, políticas e ambientais neste processo (Glanz et al., 2005). Alguns autores têm conduzido estudos sobre o ambiente obesogênico, que é caracterizado como um ambiente que inclui os fatores que influenciam as possíveis causas e efeitos que geram a obesidade, tais como o ambiente, oportunidades e condições de vida (Fisberg et al., 2016; Swinburn et al., 1999). Sabe-se que este ambiente é predominante em regiões urbanizadas, por oferecer diversas opções de estabelecimentos que comercializam alimentos calóricos e pouco nutritivos a preços mais acessíveis, como as redes de fast food, e por dispor de características que desestimulam a prática de atividade física, como a ausência ou má estruturação de parques e praças, pavimentação inapropriada de pistas e ciclovias, pouca iluminação e insegurança pública (Nascimento et al., 2015).

Atualmente, os ambientes obesogênicos tem atendido as necessidades da população, oferecendo alternativas para uma alimentação prática e rápida. Sendo assim imperativo o conhecimento destes ambientes e distribuição de estabelecimentos e espaços promotores, ou não, de saúde, para o efetivo direcionamento de políticas públicas. Até o momento, nenhum outro estudo abordou esse tema em uma capital do Nordeste, tornando-o oportuno para a condução de ações públicas de combate ao excesso de peso, que causam impacto negativo na tanto na saúde da população, quanto na economia local.

Assim, o presente estudo tem como objetivos quantificar e mapear os fatores obesogênicos de uma capital do Nordeste, por meio do conhecimento e mensuração dos estabelecimentos comerciais alimentares e dos locais para a prática de atividade física, com mapeamento da distribuição espacial de ambos, e classificação quanto ao tipo de alimentação fornecida.

\section{Metodologia}

Foi realizada pesquisa de delineamento ecológico, cartográfica, analítica observacional, de caráter quantitativo, com coleta de dados transversal de estabelecimentos comerciais de alimentação e locais para a prática de atividade física. A distribuição espacial dos estabelecimentos de comercialização de alimentos da cidade foi investigada segundo as listas telefônicas, e categorizadas em conformidade com os tipos de produtos oferecidos: (1) feira livre, (2) açougue/peixaria, (3) restaurante, (4) lojas de produtos naturais, (5) mercado/supermercado/mercearia/ frutaria, (6) padaria/delicatéssen/ conveniência, (7) pizzaria/massas, (8) cafeteria/ doceria, (9) lanchonete e (10) bar.

Posteriormente, para viabilizar as análises, estas categorias foram reagrupadas em três eixos (Quadro 1), segundo a oferta predominante de alimentos e/ou refeições. O agrupamento foi definido considerando a classificação NOVA dos alimentos segundo o Guia Alimentar para a População Brasileira (Brasil, 2014; Monteiro et al., 2010).

Quadro 1. Classificação dos estabelecimentos alimentares.

\begin{tabular}{|c|c|c|}
\hline Grupo 1 & $\begin{array}{c}\text { In natura ou minimamente } \\
\text { processados e locais que } \\
\text { produzem refeições }\end{array}$ & $\begin{array}{c}\text { (1) feira livre, (2) açougue/peixaria, (3) restaurante, (4) } \\
\text { lojas de produtos naturais }\end{array}$ \\
\hline Grupo 2 & Mistos & $\begin{array}{c}\text { (5) mercado/supermercado/mercearia/ frutaria, e } \\
\text { lanchonetes que não sejam fast food }\end{array}$ \\
\hline Grupo 3 & Processados e ultraprocessados & $\begin{array}{c}\text { (6) padaria/delicatéssen/ conveniência, (7) } \\
\text { pizzaria/massas, (8) cafeteria/ doceria, (9) lanchonete e } \\
(10) \text { bar }\end{array}$ \\
\hline
\end{tabular}

Fonte: Autores. 
Os estabelecimentos do 'Grupo 1' tiveram como critério a oferta de produtos in natura, em sua maioria. No caso de restaurantes considerou-se a preparação caseira dos alimentos in natura. O 'Grupo 2' contemplou aqueles que ofertam tanto alimentos in natura quanto processados. No caso de frutarias, observou-se que é comum que esses estabelecimentos na cidade estudada ofertem, além das frutas, produtos processados e/ou ultraprocessados, diferentemente das feiras livres. Por fim, o 'Grupo 3', os estabelecimentos que oferecem produtos e alimentos processados e ultraprocessados, em sua maioria.

Os espaços voltados à prática de atividade física e lazer foram categorizados como: (1) espaços privados, que incluíram academia, escola de dança, esportes, artes marciais e outros e (2) espaços públicos, compostos por praças, parques, ciclovias e Academias da Cidade, programa público que incentiva a prática de atividade física gratuita e acessível, no contex to dos espaços públicos. Para ambas análises foram critérios de inclusão na amostra ser estabelecimento de comercialização de alimentos ou espaço para prática de atividade física situado em uma das zonas administrativas da cidade de Aracaju (Norte, Oeste, Centro, Sul, Expansão e Santa Maria) (Prefeitura de Aracaju, 2013) e estar em funcionamento. Não foi objetivo da pesquisa avaliar comércios ambulantes.

A coleta de dados foi realizada com base na metodologia adaptada do processo de geocodificação descrito por Cayo e Talbot (Cayo \& Talbot, 2003), que consiste em obter endereços e coordenadas válidas do Sistema de Posicionamento Global (GPS), para posterior plotagem dos pontos em mapas.

O registro de endereços e coordenadas geográficas das variáveis foi realizado pelo software Google Earth (Google, 2015) em sua versão 7.1.5.1557 para Personal Computer (PC), metodologia baseada no estudo de Oreskovic et al. (Oreskovic et al., 2014).

Esses dados foram registrados em um banco de dados dividido em: (1) estabelecimentos de alimentação e (2) locais para atividade física, organizado por zonas administrativas e seus respectivos bairros, nome do estabelecimento, endereço, coordenadas, tipo de estabelecimento e classificação. Em caso de dúvidas na classificação, os locais foram visitados. Para melhor visualização da distribuição dos estabelecimentos analisados pela cidade, foram gerados mapas utilizando um Sistema de Informação Global (SIG), sistema computacional de hardware e software com informações geográficas que permitem a confecção cartográfica (Baluz, 2010).

A identificação dos estabelecimentos de comercialização de alimentos e dos espaços para prática de atividade física foi realizada mediante consulta às listas telefônicas impressas e on-line, como realizado em um estudo anterior (Glanz et al., 2007). A relação das feiras livres da cidade e das praças públicas foi acessada no site oficial da Prefeitura de Aracaju (Prefeitura de Aracaju, 2013). Os dados referentes ao Programa Academia da Cidade foram disponibilizados pela Secretaria Municipal de Saúde de Aracaju, órgão gestor responsável.

As zonas administrativas da cidade e as informações de renda domiciliar e de densidade populacional, desagregadas por bairro, foram cedidas pelo Instituto Brasileiro de Geografia e Estatística (IBGE) (Instituto Brasileiro de Geografia e Estatística, 2011). Para renda foi utilizada a classificação da Associação Brasileira de Empresas de Pesquisa (Associação Brasileira de Empresas de Pesquisa, 2014): A (de R \$ 7.800,01 a 18.800,00), B (de R \$ 4.000,01 a 7.800,00), C (de R $\$ 2.250,01$ a 4.000,00), D (de R \$ 1.350,01 a 2.250,00) e E (de R \$600,01 a 1.350,00). A densidade demográfica foi classificada em muito

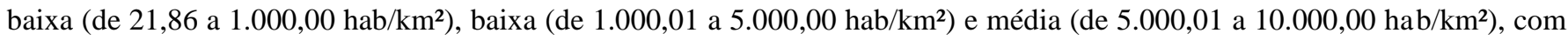
base no estudo da Prefeitura de Belo Horizonte (Prefeitura de Belo Horizonte, 2010).

A análise estatística foi realizada pelo software Statistical Package for the Social Sciences (SPSS), versão 25.0 para Windows, com frequências relativas, média e desvio padrão, valores mínimos e máximos da distribuição espacial dos estabelecimentos de alimentação e atividade física, segundo o grau de aglomeração nas zonas de Aracaju, das variáveis de renda e população. Para as variáveis categóricas foi realizado o teste Qui-quadrado de Pearson, adotando-se o nível de significância estatística de 5\% de probabilidade ( $<<0,05)$. 


\section{Resultados}

O estudo identificou 725 estabelecimentos comerciais de alimentação e 236 locais para a prática de atividade física, sendo mais frequentes os estabelecimentos de alimentação do Grupo 3 (47,6\%), e os espaços públicos para atividade física $(47,9 \%)$ (Tabela 1). Foram geradas duas cartografias: (1) distribuição espacial dos estabelecimentos de comercialização de alimentos por classes de renda média domiciliar e zona administrativa; e (3) distribuição espacial dos locais para atividade física.

Tabela 1. Tipo de estabelecimento de alimentação e locais para atividade física. Pesquisa ambiente obesogênico de uma capital do Nordeste, Brasil.

\begin{tabular}{lc}
\hline \multicolumn{1}{c}{ Variáveis } & $\%$ \\
\hline Estabelecimentos de alimentação & \\
Grupo 1 & 34,5 \\
Grupo 2 & 17,9 \\
Grupo 3 & 47,6 \\
Locais para Atividade Física & \\
Espaços públicos & 54,2 \\
Espaços privados & 45,8 \\
\hline
\end{tabular}

Grupo 1: In natura ou minimamente processados e locais que produzem refeições; Grupo 2: Mistos; Grupo 3: Processados e ultraprocessados. Fonte: Autores.

A Tabela 2 apresenta o percentual de cada grupo de estabelecimento alimentar por zona administrativa da cidade, e suas respectivas densidades populacionais e rendas domiciliares. Os estabelecimentos do Grupo 3 estão mais presentes $(27,4 \%)$ na zona de baixa densidade populacional, e os estabelecimentos do Grupo 1 mais presentes (64\%) na zona de alta renda domiciliar (Classe B), com diferença estatística significativa ( $\mathrm{p}<0,01)$ (Tabela 3).

A zona Sul apresentou maiores percentuais (Tabela 2) e aglomerados em sua porção norte, em fronteira com a zona Centro, de todas as classificações de estabelecimentos de alimentação, inclusive de feiras livres (Figura 1).

As zonas de menores densidades populacionais (Tabela 2), zona Santa Maria (2.676,73 hab/km²) e zona de Expansão (353,66 hab/km²), também são as que possuem menores aglomerações de estabelecimentos de alimentação, destacando-se a zona Santa Maria, que não apresentou nenhum estabelecimento do Grupo 3 (Figura 1). As zonas Norte e Centro tiveram uma perceptível aglomeração de estabelecimentos mistos, do Grupo 2 (31,5\%) e do Grupo 3 (16,2\%), respectivamente (Tabela 2).

No que concerne a distribuição espacial de estabelecimentos para alimentação no domicílio, as quatro categorias apresentam-se bem distribuídas entre as zonas Norte, Oeste, Sul e Centro, porém com o foco da aglomeração na zona Centro/Sul. A zona Norte também se destaca por ter considerável representação de mercado/supermercado/mercearia/frutaria e açougue/peixaria, e a zona Oeste, considerável representação de feiras livres. As zonas de Expansão e Santa Maria não apresentaram aglomerações quando comparadas às demais zonas (figura não apresentada). 
Research, Society and Development, v. 10, n. 11, e327101119578, 2021

(CC BY 4.0) | ISSN 2525-3409 | DOI: http://dx.doi.org/10.33448/rsd-v10i11.19578

Figura 1. Distribuição espacial dos estabelecimentos alimentares por grupo, segundo as classes de renda média familiar. Pesquisa ambiente obesogênico de uma capital do Nordeste, Brasil.

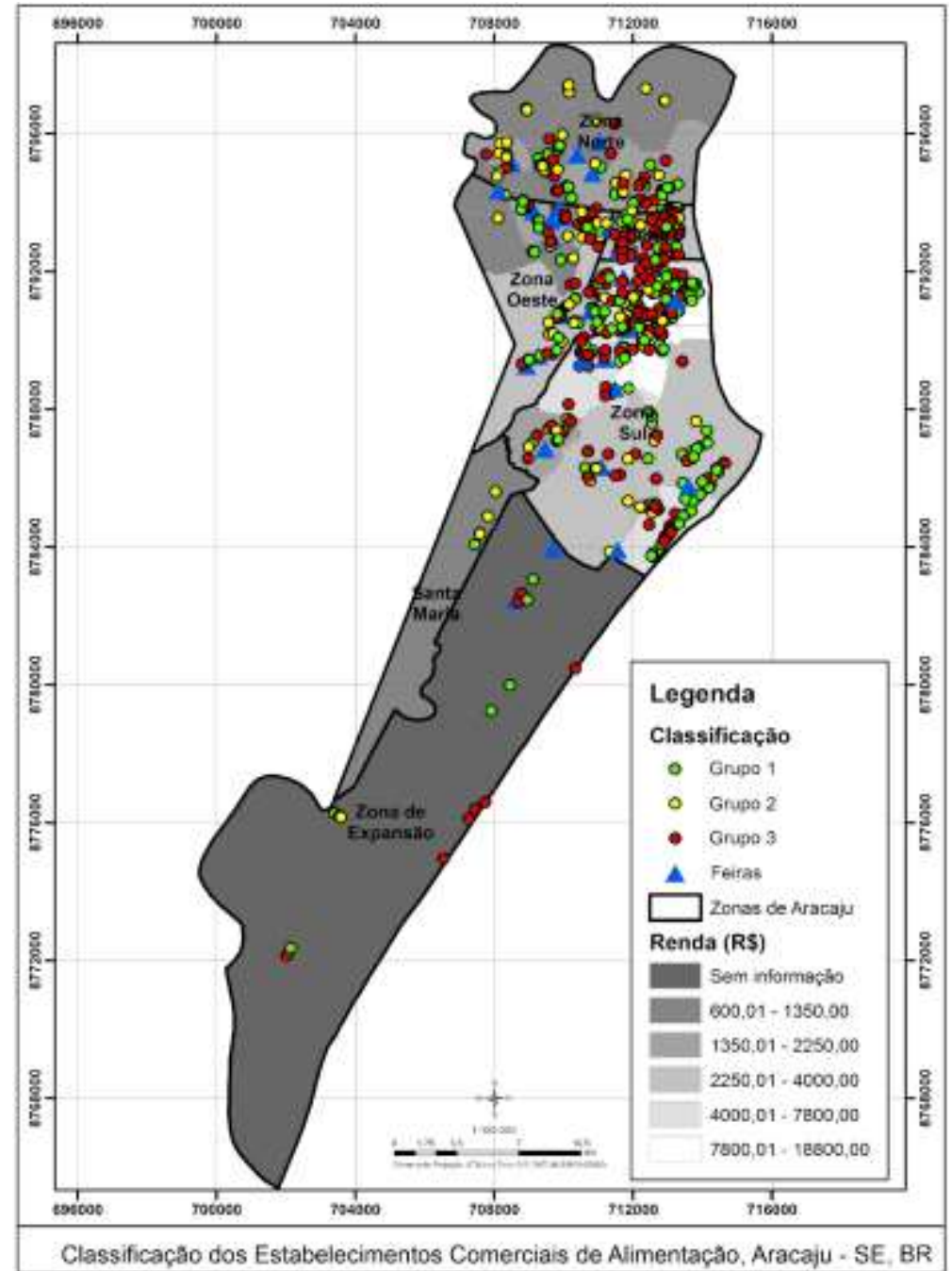

Fonte: Autores. 
Tabela 2. Tipo de estabelecimento por zonas administrativas de um capital do Nordeste, e suas respectivas densidades populacionais e rendas domiciliares. Pesquisa ambiente obesogênico, Brasil.

\begin{tabular}{|c|c|c|c|c|c|c|}
\hline \multirow{2}{*}{ Variáveis } & \multicolumn{6}{|c|}{$\%$ por zonas administrativas } \\
\hline & Norte & Oeste & Centro & Sul & Expansão & $\begin{array}{l}\text { Santa } \\
\text { Maria }\end{array}$ \\
\hline \multicolumn{7}{|l|}{$\begin{array}{l}\text { Estabelecimentos de } \\
\text { Alimentação }\end{array}$} \\
\hline Grupo 1 & 10,8 & 14,8 & 8,4 & 62,0 & 3,2 & 0,8 \\
\hline Grupo 2 & 31,5 & 19,2 & 7,7 & 38,5 & 1,5 & 1,5 \\
\hline Grupo 3 & 8,7 & 14,8 & 16,2 & 57,7 & 2,6 & 0,0 \\
\hline \multicolumn{7}{|l|}{$\begin{array}{c}\text { Locais para Atividade } \\
\text { Física }\end{array}$} \\
\hline $\begin{array}{l}\text { Espaços } \\
\text { Públicos }\end{array}$ & 14,1 & 18,8 & 11,7 & 52,3 & 1,6 & 1,6 \\
\hline $\begin{array}{l}\text { Espaços } \\
\text { Privados }\end{array}$ & 6,5 & 15,7 & 10,2 & 62,0 & 4,6 & 0,9 \\
\hline $\begin{array}{c}\text { Densidade Populacional } \\
\left(\mathrm{hab} / \mathrm{km}^{2}\right)^{1} \\
\text { Renda domiciliar }(\mathrm{R} \$) \\
1,2\end{array}$ & $6.455,4$ & $6.333,2$ & $5.238,6$ & $4.828,2$ & 353,6 & $2.676,7$ \\
\hline Média & $1.322,52$ & $1.975,28$ & $3.079,35$ & $5.602,63$ & SI & $843,38^{3}$ \\
\hline Desvio-padrão & $\pm 388,72$ & $\pm 878,66$ & $\pm 886,93$ & $\pm 3.180,92$ & SI & - \\
\hline Mínimo & 814,54 & 943,24 & $2.106,31$ & $2.053,18$ & SI & - \\
\hline Máximo & $10.708,17$ & $3.394,51$ & $3.842,53$ & $13.852,16$ & SI & - \\
\hline
\end{tabular}

Nota. Estabelecimentos de alimentação: $\mathrm{n}=725$; locais para a prática de atividade física: $\mathrm{n}=236$.

${ }^{1}$ Censo Demográfico 2010 (IBGE, 2012). ${ }^{2}$ Cálculo a partir da renda desagregada por bairros. ${ }^{3}$ Zona administrativa com único bairro, sem possibilidade de cálculo da média. SI = sem informação. Grupo 1: In natura ou minimamente processados e locais que produzem refeições; Grupo 2: Mistos; Grupo 3: Processados e ultraprocessados. Fonte: Autores.

As distribuições espaciais dos estabelecimentos de alimentação relacionadas à renda média familiar de cada zona (Figura 1) mostra que a aglomeração desses estabelecimentos está situada na área de maiores rendas e elevadas densidades populacionais (Tabela 2): zonas Sul, Centro, Norte e Oeste. As zonas de menores rendas têm também menores representações de estabelecimentos e menores densidades populacionais: zonas de Expansão e Santa Maria.

A distribuição espacial dos locais para atividade física está apresentada na Figura 2, os espaços públicos foram representados na forma de espaços públicos e academia da cidade. A zona Sul apresentou o maior percentual (Tabela 2) de aglomeração desses locais, seguida das zonas Norte, Oeste e Centro. Essas mesmas zonas são cercadas pelos aglomerados de estabelecimentos comerciais de alimentação. As zonas de Expansão e Santa Maria também apresentaram as menores aglomerações de locais para atividade física. As Academias da Cidade apresentaram dispersão pela cidade, ausentes apenas nas zonas Centro e Expansão. 
Figura 2. Distribuição espacial de locais para atividade física. Pesquisa ambiente obesogênico de uma capital do Nordeste, Brasil.

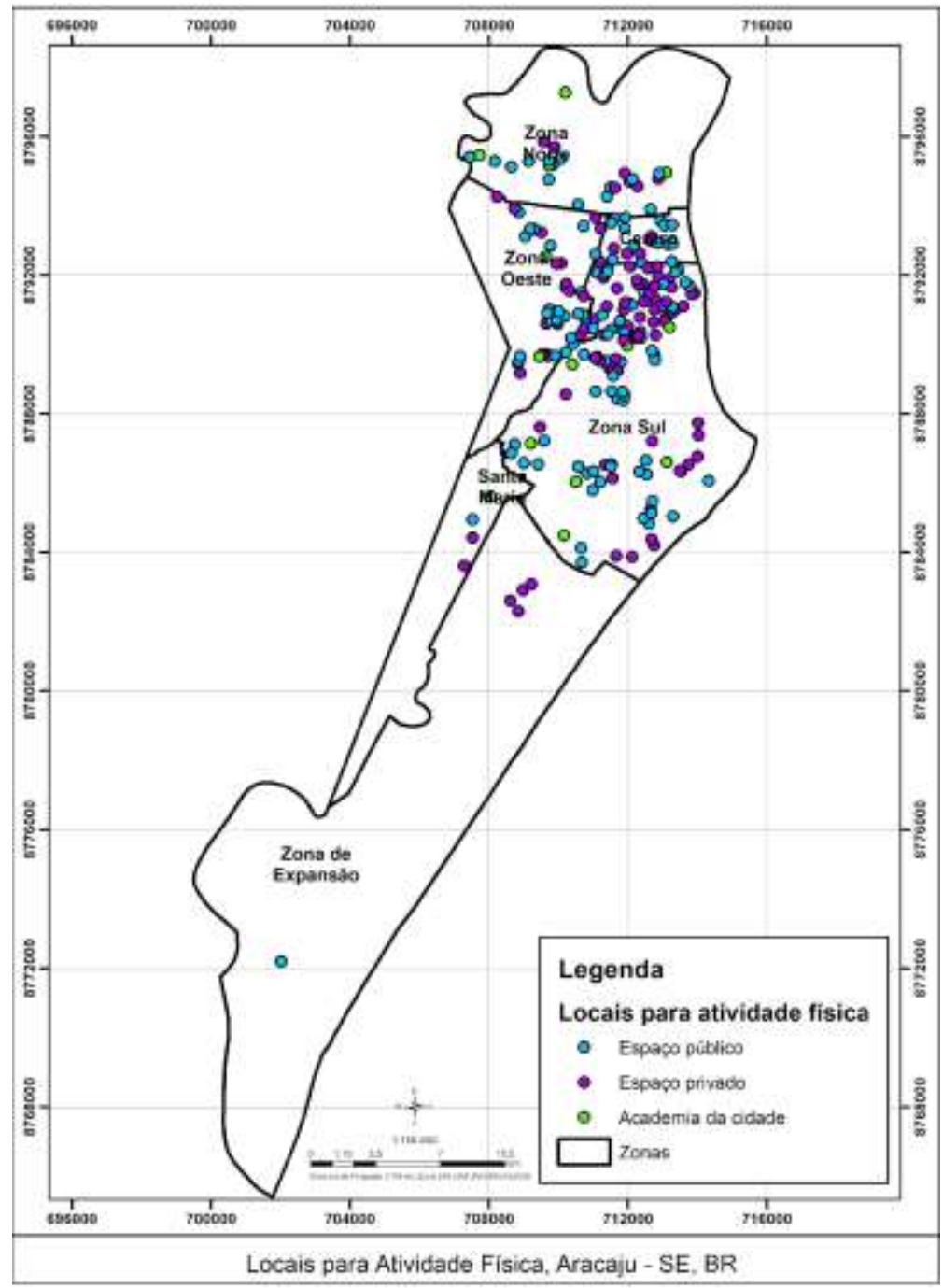

Fonte: Autores.

Dentro dos espaços públicos, as academias também foram analisadas separadamente com o intuito de identificar se a presença deste equipamento de saúde seria diferente das demais categorias analisadas nas diferentes regiões da cidade. Os resultados mostraram que os espaços públicos para atividade física $(61,6 \%)$ estão mais presentes nas zonas de média densidade populacional, os espaços privados $(71,4 \%)$ na zona de muito baixa densidade e as academias da cidade $(6,6 \%)$ nas zonas de baixa densidade, com diferença estatística não significativa $(\mathrm{p}=0,26)$. As três categorias mostraram-se mais presentes nas zonas de baixa densidade populacional $(58,1 \%)$, e de alta de renda domiciliar (Classe B) (56,8\%). As academias da cidade se concentraram (43\%) nas zonas de baixa e muito baixa renda (classes D e E, respectivamente), com diferença estatística não significativa $(\mathrm{p}=0,07)$.

\section{Discussão}

No presente estudo, observou-se maior exposição da população de Aracaju a estabelecimentos de alimentos ultraprocessados, mas também a espaços públicos para atividade física. A disponibilidade e o acesso aos alimentos, como também, às oportunidades para a prática de atividade física em um ambiente são considerados fatores chave para entender a 
influência do ambiente nas escolhas alimentares e prática de atividade física, fatores fortemente associados à obesidade vivenciada em âmbito global (Mendes, 2012; Swinburn et al., 2013; Swinburn et al., 2019; Swinburn et al., 1999).

No geral, a concentração de estabelecimentos em uma região é determinada pela renda, sendo assim, quanto maior a renda da região maior o número de estabelecimentos alimentares presentes nela (Black \& Macinko, 2008; Duran et al., 2016). Sabe-se também que os centros urbanos são áreas onde a dinâmica do capital e o fluxo de pessoas são mais intensos e, consequentemente, áreas de concentração de atividades comerciais e serviços (Soeiro et al., 2014). As zonas Centro e Sul são os centros urbanos e comerciais de Aracaju, sendo aquelas com maiores aglomerados de estabelecimentos alimentares, com uma alta concentração de estabelecimentos do Grupo 3. Sendo assim, são os ambientes mais obesogênicos da cidade. Estudos anteriores demonstraram uma associação entre disponibilidade de estabelecimentos não saudáveis com o consumo e comportamento alimentar de risco da população (Giskes et al., 2011; Stephens et al., 2011; Vaughan et al., 2017), estes resultados contrastam com os achados de um estudo realizado no Rio de Janeiro, o qual demonstrou que quanto maior o número de estabelecimentos comerciais, independentemente do tipo, maiores as chances de um maior consumo de frutas e vegetais folhosos da população (Curioni et al., 2020). Porém, no geral, sabe-se que a alta disponibilidade de estabelecimentos não saudáveis é considerada fator de risco para o ganho de peso e prevalência de sobrepeso e obesidade (Bodor et al., 2010; Mehta \& Chang, 2008; Morland \& Evenson, 2009).

$\mathrm{O}$ ambiente alimentar vem sendo homogeneizado pelas grandes cadeias de lanchonetes, em que os estabelecimentos do tipo fast food e as comidas industrializadas estão cada vez mais presentes no cotidiano da população, tendo a capacidade de afetar a saúde por modular comportamentos e escolhas não saudáveis (Nascimento et al., 2015; Sichieri \& Souza, 2008). Aracaju é uma capital em expansão, e a sua população com o ritmo de vida cada vez mais acelerado tende a optar por refeições mais práticas que atendam a esta dinamicidade. Apesar do grande número de opções de estabelecimentos do Grupo 1, principalmente nas zonas Sul e Norte, observou-se um número expressivo de opções do Grupo 3 por toda cidade, com destaque para a presença desses estabelecimentos nas áreas de menor densidade demográfica

Sabe-se que bairros periféricos e com baixos indicadores sociais geralmente apresentam um acesso inadequado aos alimentos (Duran et al., 2013), fazendo com que os residentes precisem se deslocar até regiões centrais que apresentam melhor disponibilidade de alimentos in natura ou minimamente processados, o que dificulta o consumo dos mesmos. No presente estudo, foi observada uma maior exposição dos moradores dessas zonas (Santa Maria e Expansão) a estabelecimentos dos Grupos 2 e 3, o que pode ocasionar o deslocamento para outras regiões em busca de estabelecimentos do Grupo 1 ou o consumo de alimentos processados e ultraprocessados. O acesso aos alimentos é fundamental para mudança de hábitos alimentares, principalmente na população com menor poder aquisitivo, uma vez que baixa renda é um fator associado a comportamentos alimentares obesogênicos (Brasil, 2018; Giskes et al., 2011).

A categoria mista de estabelecimentos, Grupo 2, esteve mais dispersa pela cidade. Este achado corrobora com os resultados de um estudo realizado na cidade de São Paulo, onde esta categoria foi a mais dispersa e aglomerada em determinadas regiões dos distritos estudados (Duran et al., 2015). Já em outro estudo também realizado na cidade de São Paulo os mercados especializados em frutas e verduras (frutarias), assim como em Aracaju, estão mais disponíveis em áreas de maior nível socioeconômico (Jaime et al., 2011). Diferente do encontrado em estudo realizado em Belo Horizonte, onde estes estabelecimentos apresentaram uma distribuição espacial variada e com pouca aglomeração, o que permite à maior parte da população da cidade o acesso físico a uma opção mais saudável de alimentação (Mendes, 2012).

As feiras livres são espaços de comercialização de alimentos, inclusive da agricultura familiar, que valorizam a economia local e apresentam menores preços, quando comparadas aos demais comércios varejistas, além da valorização social e da troca de saberes entre feirantes e consumidores (Godoy \& Anjos, 2007). Em Aracaju, as feiras acontecem em todas as zonas administrativas, o que representa incentivo do governo à economia local e à oferta de alimentos mais saudáveis. Porém, 
a quantidade de estabelecimentos do Grupo 2 ainda é muito maior, além de serem estabelecimentos fixos, que estão sempre abertos, quando comparados às feiras livres que acontecem, no máximo, duas vezes na semana. A busca da população por praticidade faz com que esse tipo de estabelecimento seja bastante procurado para a aquisição de alimentos, que apesar de comercializarem alimentos in natura divide espaço com alimentos ultraprocessados, acompanhados de propagandas e promoções (Brasil, 2014)

Sendo assim, os estabelecimentos do tipo fast food, encontram no cenário contemporâneo uma oportunidade de venda de seus produtos ultraprocessados, com preços geralmente inferiores aos alimentos processados ou in natura, que alteram os hábitos alimentares locais. Ações como essas retiram a identidade cultural do local e geram ambientes alimentares obesogênicos (Monteiro et al., 2018). No entanto, estes produtos ocultam em seus preços um custo mais elevado: a obesidade e as doenças crônicas não transmissíveis (DCNTs), que comprometem a qualidade de vida e capacidade de trabalho, o que pode onerar os serviços públicos de forma trágica.

A prática de atividade física é umas das formas de prevenção e tratamento da obesidade e DCNTs (Warburton \& Bredin, 2017; World Health Organization, 2004). Em Aracaju há muitas opções para esta prática, espaços privados como academias e escolas de dança e lutas estão espalhados pelas zonas de maiores rendas e densidades populacionais, assim como os espaços públicos. Além disso, o Programa Academia da Cidade, incentivo do poder público para a prática de atividade física, com aulas gratuitas direcionadas nas praças da cidade, está disperso pelas zonas de maiores densidades populacionais, mas ainda em menor quantidade.

Porém, no Brasil, um estudo relatou que apenas $28,3 \%$ dos indivíduos relataram a presença de instalações públicas de lazer ou de baixo custo em sua vizinhança (Sallis et al., 2009) e em outro estudo nacional, 56\% dos entrevistados relataram ter conhecimento sobre os locais públicos de esporte e lazer (Agrizzi et al., 2021). Sabe-se que há resultados positivos à saúde da população quando há espaços urbanos públicos propícios para a prática de atividade física, como demonstrado no estudo de Durand et al., onde a presença de praças e de parques foi relacionada a maiores níveis de atividade física no lazer e, consequentemente, menores taxas de sobrepeso e obesidade (Durand et al., 2011). Outro estudo demonstrou uma correlação negativa entre sobrepeso e a maior densidade de parques e espaços públicos para prática de atividade física (Jaime et al., 2011).

Há que se considerar também que a capital sergipana conta com uma orla de $6 \mathrm{~km}$ de extensão, com opções de ambientes naturais e construídos para a prática de atividade física, além de $35 \mathrm{~km}$ de praia, um calçadão na zona Sul com $1 \mathrm{~km}$ de extensão e mais de 60 km de ciclovias (Prefeitura de Aracaju., 2019; Superintendência Municipal de Transportes e Trânsito, 2016). Uma pesquisa realizada em Belo Horizonte que estudou os espaços públicos para atividade física, mostrou que a capital mineira conta com 107 locais georreferenciados para essa finalidade (Mendes, 2012). Belo Horizonte é expressivamente maior que Aracaju, que conta com 128 locais públicos, sem considerar a orla e as ciclovias.

Apesar da disponibilidade e acesso gratuito, 44,9\% da população aracajuana está insuficientemente ativa (Brasil, 2020), isso significa que a mesma população exposta a um ambiente alimentar obesogênico também é exposta a um ambiente protetor da saúde, porém, não necessariamente essa população irá usufruir integralmente desses espaços para prática de atividade física. Isso pode ocorrer devido a questões individuais ou por questões de ambiência, uma vez que esses ambientes podem ser, muitas vezes, inseguros e perigosos, e apresentarem barreiras estruturais, como iluminação insuficiente das ruas, trânsito intenso, e calçadas e instalações recreativas inadequadas, o que reduz as taxas de atividade física ao ar livre (Mendes, 2012; Nascimento et al., 2015). E de fato, um estudo nacional demonstrou que o maior conhecimento de locais públicos para prática de atividade física foi observado em cidades onde foram avaliadas melhores condições ambientais urbanas (Agrizzi $e t$ al., 2021). 
Essa dupla exposição faz com que as decisões individuais sejam um fator determinante para proteção da obesidade, mas não são suficientes para a garantia de práticas alimentares e de atividade física em âmbito coletivo. Com isso, só serão efetivas as ações que integrarem medidas individuais e medidas dirigidas ao ambiente em que se inserem a população (Castro, 2015). Cabe ainda ressaltar que o consumo alimentar não saudável fere o direito humano à alimentação adequada e saudável, e não promove a segurança alimentar e nutricional da população, que é dever do poder público, segundo a Lei Orgânica de Segurança Alimentar e Nutricional do Brasil (Brasil, 2006). Segundo Batista (2008), "O ambiente obesogênico não é de um fatalismo irreversível", mas é necessário conhecer os ambientes e propor políticas públicas e estratégias que sejam tão agressivas quanto é a expansão das influências obesogênicas (Batista Filho, 2008; Sichieri \& Souza, 2008).

$\mathrm{O}$ presente estudo apresentou limitações quanto à coleta de dados, uma vez que a fonte utilizada para identificar os estabelecimentos comerciais de alimentação foi não governamental e utilizada de forma isolada. A utilização de listas telefônicas como base de dados, apesar da praticidade, pode não representar a realidade do ambiente comunitário sendo potencial viés pela dinâmica mudança ambiental durante o tempo, sendo necessários recursos financeiros e logísticos para coleta in loco. Além disso, a classificação dos estabelecimentos não garante o tipo de alimentos vendidos, o que pode ter resultado em agrupamento inapropriado, tornando pertinente o estudo do ambiente alimentar do consumidor na cidade. Porém, este é o primeiro estudo a avaliar o ambiente obesogênico de uma capital do Nordeste, o que o torna relevante para o estudo do ambiente comunitário do país e para análise de políticas públicas locais que incentivem os hábitos de vida saudáveis.

\section{Considerações Finais}

O ambiente obesogênico de Aracaju-SE encontra-se concentrado no Centro/Sul, área mais desenvolvida da cidade, com moderada dispersão desse ambiente pelas demais áreas em crescimento. Os fatores protetores da saúde contra a obesidade, como estabelecimentos saudáveis de alimentação e locais para a prática de atividade física, apresentam a mesma distribuição espacial com concentração no Centro/Sul. Assim, a população está exposta tanto ao ambiente promotor da obesidade quanto ao estilo de vida saudável, cabendo ao poder público propostas de controle complementares mediante a regulação das propagandas de alimentação não saudável, taxação ou subsídios de determinados alimentos e regulamentação da composição de alimentos ultraprocessados.

Apesar da concentração de espaços para prática de exercício físico ser bem distribuída pela cidade, a população tornase mais vulnerável ao ambiente alimentar obesogênico devido às barreiras encontradas para a adoção do estilo de vida saudável. Mais estudos são necessários para identificar as barreiras percebidas pela população, o que auxiliará a compreender os altos índices de inatividade física atualmente.

Estratégias que incentivem e facilitem as escolhas saudáveis, como ações educativas, e que protejam os indivíduos de fatores ambientais que levam a escolhas não saudáveis, podem ser tomadas por meio de políticas públicas para reverter o quadro de maior exposição ao ambiente obesogênico. $\mathrm{O}$ incentivo à prática de atividade física, como um fator de qualidade de vida e protetor contra o desenvolvimento da obesidade, deve ser realizado através da melhoria da estrutura e da segurança dos espaços públicos, bem como de incentivo e orientação da população.

\section{Agradecimentos}

Este estudo foi financiado em parte pela Fundação de Apoio à Pesquisa e a Inovação Tecnológica do Estado de Sergipe (Fapitec/SE) - Brasil e Coordenação de Aperfeiçoamento de Pessoal de Nível Superior - Brasil (CAPES - Código de financiamento 001). 


\section{Referências}

Agrizzi, P., Dourado, T. E. P. S., Silva, J. I. da, \& Andrade, A. C. de S. (2021). Fatores associados ao conhecimento de locais públicos de esporte e lazer nas capitais brasileiras. Revista Brasileira de Atividade Física \& Saúde, 26, 1-8. https://doi.org/10.12820/rbafs.26e0201

Associação Brasileira de Empresas de Pesquisa. (2014). Critério Brasil 2014. ABEP. file:///C:/Users/marce/Downloads/09_cceb_2014.pdf

Baluz, R. A. . (2010). Geoprocessamento aliado à técnica de data warehouse como ferramenta para auxílio na saúde pública. Revista F@pciência, ApucaranaPR, ISSN 1984-2333, 103-116.

Batista Filho, M. (2008). Debate sobre o artigo de Sichieri \& Souza. Cadernos de Saúde Pública. https://doi.org/10.1590/s0102-311x2008001400005

Black, J. L., \& Macinko, J. (2008). Neighborhoods and obesity. Nutrition Reviews, 66(1), 2-20. https://doi.org/10.1111/j.1753-4887.2007.00001.x

Bodor, J. N., Rice, J. C., Farley, T. A., Swalm, C. M., \& Rose, D. (2010). The Association between Obesity and Urban Food Environments. Journal of Urban Health, 87(5), 771-781. https://doi.org/10.1007/s11524-010-9460-6

Brasil. (2006). Lei N 11.346, DE 15 De Setembro De 2006. Cria o Sistema Nacional de Segurança Alimentar e Nutricional - SISAN com vistas em assegurar o direito humano à alimentação adequada e dá outras providências.

Brasil. (2018). Mapeamento dos Desertos Alimentares no Brasil. https://aplicacoes.mds.gov.br/sagirmps/noticias/arquivos/files/Estudo_tecnico_mapeamento _desertos_alimentares.pdf

Brasil, M. da S. (2014). Guia alimentar para a população brasileira (2. ed.).

Brasil, M. da S. (2020). Vigitel Brasil 2019: vigilância de fatores de risco e proteção para doenças crônicas por inquérito telefônico : estimativas sobre frequência e distribuição sociodemográfica de fatores de risco e proteção para doenças crônicas nas capitais dos 26 estados (Ministério da Saúde (ed.)). https://bvsms.saude.gov.br/bvs/publicacoes/vigitel_brasil_2019_vigilancia_fatores_risco.pdf

Castro, I. R. R. de. (2015). Desafios e perspectivas para a promoção da alimentação adequada e saudável no Brasil. Cad. Saúde Pública. https://doi.org/10.1021/jp808453z

Cayo, M. R., \& Talbot, T. O. (2003). Positional error in automated geocoding of residential addresses. International Journal of Health Geographics. https://doi.org/10.1186/1476-072X-2-10

Cockerham, W. C., Hamby, B. W., \& Oates, G. R. (2017). The Social Determinants of Chronic Disease. American Journal of Preventive Medicine, 52(1), S5S12. https://doi.org/10.1016/j.amepre.2016.09.010

Curioni, C. C., Boclin, K. L. S., Silveira, I. H., Canella, D. S., Castro, I. R. R., Bezerra, F. F., Junger, W., \& Faerstein, E. (2020). Neighborhood food environment and consumption of fruit and leafy vegetables: Pro-Saude Study, Brazil. Public Health, 182, 7-12. https://doi.org/10.1016/j.puhe.2020.01.004

Duran, A. C., de Almeida, S. L., Latorre, M. do R. DO, \& Jaime, P. C. (2016). The role of the local retail food environment in fruit, vegetable and sugarsweetened beverage consumption in Brazil. Public Health Nutrition, 19(6), 1093-1102. https://doi.org/10.1017/S1368980015001524

Duran, A. C. F. L. (2013). Ambiente alimentar urbano em São Paulo, Brasil: avaliação, desigualdades e associação com consumo alimentar [Universidade de São Paulo]. https://doi.org/10.11606/T.6.2013.tde-02102013-164136

Duran, A. C., Lock, K., Latorre, M. do R. D. O., \& Jaime, P. C. (2015). Evaluating the use of in-store measures in retail food stores and restaurants in Brazil. Revista de Saude Publica. https://doi.org/10.1590/S0034-8910.2015049005420

Durand, C. P., Andalib, M., Dunton, G. F., Wolch, J., \& Pentz, M. A. (2011). A systematic review of built environment factors related to physical activity and obesity risk: implications for smart growth urban planning. Obesity Reviews, 12(5), e173-e182. https://doi.org/10.1111/j.1467-789X.2010.00826.x

Fisberg, M., Maximino, P., Kain, J., \& Kovalskys, I. (2016). Obesogenic environment - intervention opportunities. Jornal de Pediatria, 92(3), S30-S39. https://doi.org/10.1016/j.jped.2016.02.007

Giskes, K., van Lenthe, F., Avendano-Pabon, M., \& Brug, J. (2011). A systematic review of environmental factors and obesogenic dietary intakes among adults: are we getting closer to understanding obesogenic environments? Obesity Reviews, 12(5), e95-e106. https://doi.org/10.1111/j.1467-789X.2010.00769.x

Glanz, K., Sallis, J. F., Saelens, B. E., \& Frank, L. D. (2005). Healthy nutrition environments: Concepts and measures. American Journal of Health Promotion, 19(5), 330-333. https://doi.org/10.4278/0890-1171-19.5.330

Glanz, K., Sallis, J. F., Saelens, B. E., \& Frank, L. D. (2007). Nutrition Environment Measures Survey in Stores (NEMS-S). Development and Evaluation. American Journal of Preventive Medicine. https://doi.org/10.1016/j.amepre.2006.12.019

Godoy, W. I., \& Anjos, F. S. dos. (2007). A Importância das Feiras Livres Ecológicas: Um Espaço de Trocas e Saberes da Economia Local. Revista Brasileira de Agroecologia.

Google. (2015). Google Earth. (Version 7.1.5.1557) [Computer Program].

Instituto Brasileiro de Geografia e Estatística. (2011). Censo Demográfico 2010: Características da população e dos domicílios - Resultados do universo. In Instituto Brasileiro de Geografia e Estatística. https://doi.org/0104-3145

Instituto Brasileiro de Geografia e Estatística. (2020a). Pesquisa nacional de saúde: 2019: atenção primária à saúde e informações antropométricas : Brasil / IBGE, Coordenação de Trabalho e Rendimento, [Ministério da Saúde] (IBGE (ed.)). https://biblioteca.ibge.gov.br/visualizacao/livros/liv101758.pdf 
Instituto Brasileiro de Geografia e Estatística. (2020b). Pesquisa nacional de saúde: 2019: percepção do estado de saúde, estilos de vida, doenças crônicas e saúde bucal: Brasil e grandes regiões / IBGE, Coordenação de Trabalho e Rendimento, [Ministério da Saúde] (IBGE (ed.)). https://biblioteca.ibge.gov.br/visualizacao/livros/liv101764.pdf

Jaime, P. C., Duran, A. C., Sarti, F. M., \& Lock, K. (2011). Investigating Environmental Determinants of Diet, Physical Activity, and Overweight among Adults in Sao Paulo, Brazil. Journal of Urban Health, 88(3), 567-581. https://doi.org/10.1007/s11524-010-9537-2

Mehta, N. K., \& Chang, V. W. (2008). Weight Status and Restaurant Availability. American Journal of Preventive Medicine, 34(2), 127-133. https://doi.org/10.1016/j.amepre.2007.09.031

Mendes, L. L. (2012). Ambiente construído e ambiente social - associações com o excesso de peso em adultos. Universidade Federal de Minas Gerais, Belo Horizinte, $M G$.

Monteiro, C. A., Cannon, G., Moubarac, J.-C., Levy, R. B., Louzada, M. L. C., \& Jaime, P. C. (2018). The UN Decade of Nutrition, the NOVA food classification and the trouble with ultra-processing. Public Health Nutrition, 21(01), 5-17. https://doi.org/10.1017/S1368980017000234

Monteiro, C. A., Levy, R. B., Claro, R. M., Castro, I. R. R. de, \& Cannon, G. (2010). A new classification of foods based on the extent and purpose of their processing. Cadernos de Saúde Pública, 26(11), 2039-2049. https://doi.org/10.1590/S0102-311X2010001100005

Morland, K. B., \& Evenson, K. R. (2009). Obesity prevalence and the local food environment. Health \& Place, 15(2), 491-495. https://doi.org/10.1016/j.healthplace.2008.09.004

Nascimento, M. A. S., Zucolotto, D. C. C., \& Sartorelli, D. S. (2015). Associação entre a percepção de atributos ambientais e excesso de peso: um estudo realizado em um município de pequeno porte. Cadernos de Saúde Pública, 31(1), 173-182. https://doi.org/10.1590/0102-311X00015814

Nilson, E. A. F., Andrade, R. da C. S., Brito, D. A. de, \& Michele Lessa de, O. (2020). Custos atribuíveis a obesidade, hipertensão e diabetes no Sistema Único de Saúde, Brasil, 2018. Revista Panamericana de Salud Pública, 44, 1. https://doi.org/10.26633/RPSP.2020.32

Oreskovic, N. M., Blossom, J., Robinson, A. I., Chen, M. L., Uscanga, D. K., \& Mendoza, J. A. (2014). The influence of the built environment on outcomes from a "walking school bus study": A cross-sectional analysis using geographical information systems. Geospatial Health. https://doi.org/10.4081/gh.2014.4

Paim, J. S. (2012). Determinantes sociais da saúde. Almanaque Dant, 7.

Prefeitura de Aracaju. (2013). Feiras Livres e Praças de Aracaju.

Prefeitura de Aracaju. (2019). Praia é opção de lazer durante o verão. https://www.aracaju.se.gov.br/financas/index.php?act=leitura\&codigo=48888

Prefeitura de Aracaju. (2008). Globo Repórter classifica Aracaju como a capital brasileira da qualidade de vida. Http://Www.Aracaju.Se.Gov.Br/Index.Php?Act=leitura\&codigo=34646.

Prefeitura de Aracaju. (2013). Consulta fotográfica de Aracaju: zonas administrativas. www.Aracaju.Se.Gov.Br/Mapas_Aracaju.Pps.

Prefeitura de Belo Horizonte. (2010). Densidade demográfica por bairro em Belo Horizonte - 2010. Belo Horizinte.

Sallis, J. F., Bowles, H. R., Bauman, A., Ainsworth, B. E., Bull, F. C., Craig, C. L., Sjöström, M., De Bourdeaudhuij, I., Lefevre, J., Matsudo, V., Matsudo, S., Macfarlane, D. J., Gomez, L. F., Inoue, S., Murase, N., Volbekiene, V., McLean, G., Carr, H., Heggebo, L. K., \& Bergman, P. (2009). Neighborhood Environments and Physical Activity Among Adults in 11 Countries. American Journal of Preventive Medicine, 36(6), 484-490. https://doi.org/10.1016/j.amepre.2009.01.031

Sichieri, R., \& Souza, R. A. de. (2008). As autoras respondem. Cadernos de Saúde Pública. https://doi.org/10.1590/s0102-311x2008001400008

Soeiro, Í. C. de M., Ferreira, S. N. B. de A., \& Brasileiro, R. S. (2014). Qualidade de vida e urbanização: uma análise a partir do centro da cidade do Recife, PE. Revistas Movimentos Sociais e Dinâmicas Espaciais, 3(1), 68-85.

Stephens, L. D. A., McNaughton, S. A., Crawford, D., MacFarlane, A., \& Ball, K. (2011). Correlates of dietary resilience among socioeconomically disadvantaged adolescents. European Journal of Clinical Nutrition, 65(11), 1219-1232. https://doi.org/10.1038/ejcn.2011.107

Superintendência Municipal de Transportes e Trânsito. (2016). Prefeitura recupera trechos danificados ciclovias. Aracaju.

Swinburn, B., Kraak, V. I., Allender, S., Atkins, V. J., Baker, P. I., Bogard, J. R., Brinsden, H., Calvillo, A., De Schutter, O., Devarajan, R., Ezzati, M., Friel, S., Goenka, S., Hammond, R. A., Hastings, G., Hawkes, C., Herrero, M., Hovmand, P. S., Howden, M., ... Dietz, W. H. (2019). The Global Syndemic of Obesity, Undernutrition, and Climate Change: The Lancet Commission report. The Lancet, 393(10173), 791-846. https://doi.org/10.1016/S0140$6736(18) 32822-8$

Swinburn, B., Sacks, G., Vandevijvere, S., Kumanyika, S., Lobstein, T., Neal, B., Barquera, S., Friel, S., Hawkes, C., Kelly, B., L’Abbé, M., Lee, A., Ma, J., Macmullan, J., Mohan, S., Monteiro, C., Rayner, M., Sanders, D., Snowdon, W., \& Walker, C. (2013). INFORMAS (International Network for Food and Obesity/non-communicable diseases Research, Monitoring and Action Support): overview and key principles. Obesity Reviews, 14, 1-12. https://doi.org/10.1111/obr.12087

Swinburn, B., Egger, G., \& Raza, F. (1999). Dissecting Obesogenic Environments: The Development and Application of a Framework for Identifying and Prioritizing Environmental Interventions for Obesity. Preventive Medicine, 29(6), 563-570. https://doi.org/10.1006/pmed.1999.0585

Vaughan, C. A., Collins, R., Ghosh-Dastidar, M., Beckman, R., \& Dubowitz, T. (2017). Does where you shop or who you are predict what you eat?: The role of stores and individual characteristics in dietary intake. Preventive Medicine, 100, 10-16. https://doi.org/10.1016/j.ypmed.2017.03.015

Warburton, D. E. R., \& Bredin, S. S. D. (2017). Health benefits of physical activity: A systematic review of current systematic reviews. Current Opinion in 
Research, Society and Development, v. 10, n. 11, e327101119578, 2021

(CC BY 4.0) | ISSN 2525-3409 | DOI: http://dx.doi.org/10.33448/rsd-v10i11.19578

Cardiology, 32(5), 541-556. https://doi.org/10.1097/HCO.0000000000000437

World Health Organization, (WHO). (2004). Global strategy on diet, physical activity and health. In Scandinavian Journal of Nutrition/Naringsforskning. https://doi.org/10.1080/11026480410034349

World Health Organization, (WHO). (2021). Obesity and overweight. https://www.who.int/en/news-room/fact-sheets/detail/obesity-and-overweight 